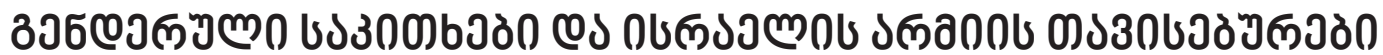

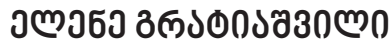

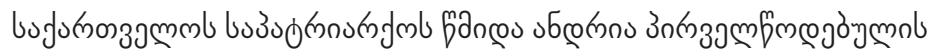

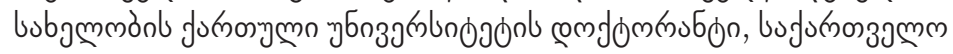

https://doi.org/10.35945/gb.2017.04.017

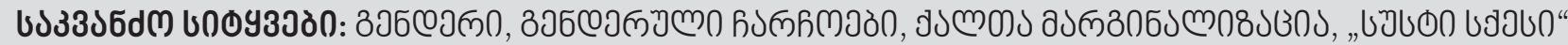

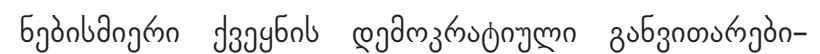

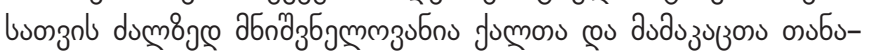

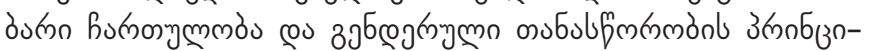

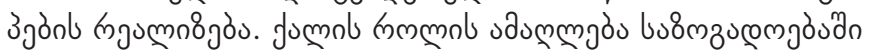

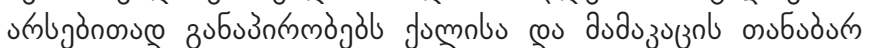

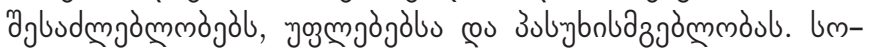

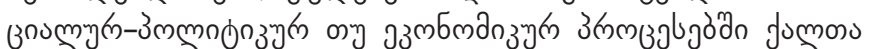

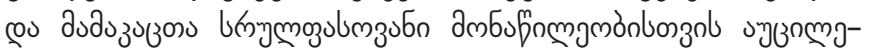

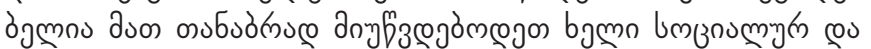

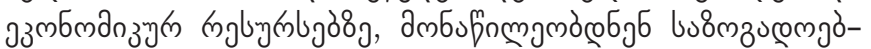

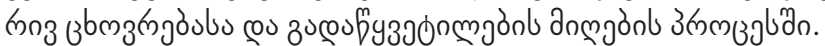

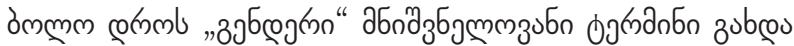

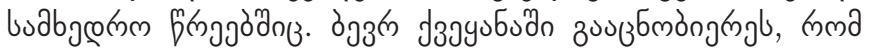

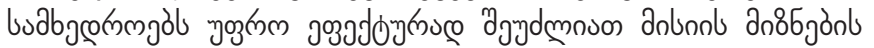

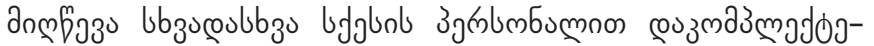

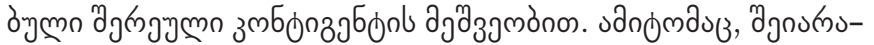

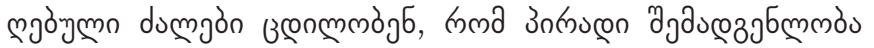

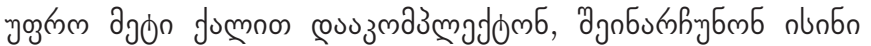

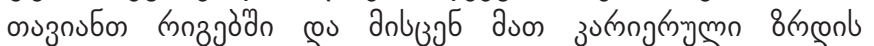

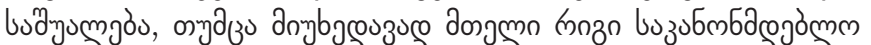

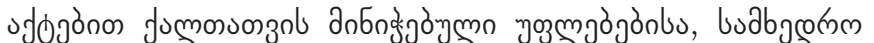

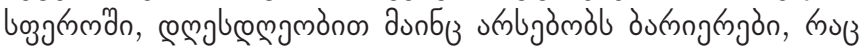

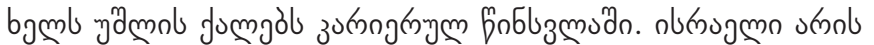

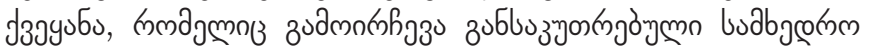

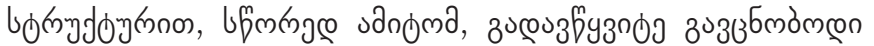

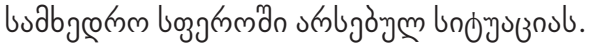

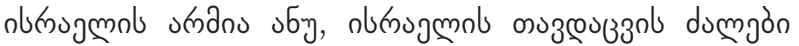

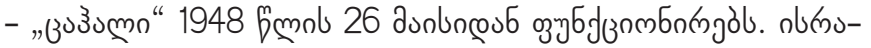

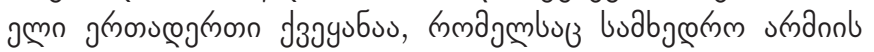

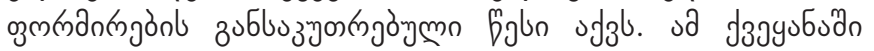

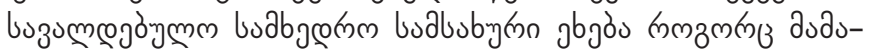

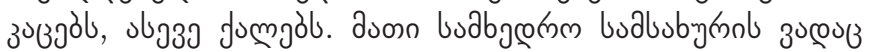
zublu8

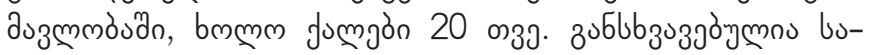

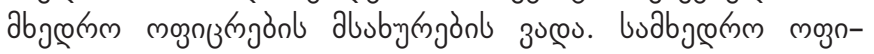

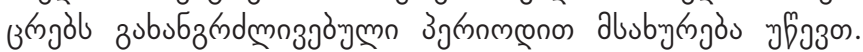

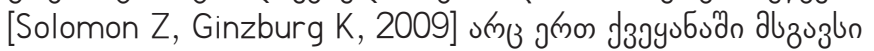

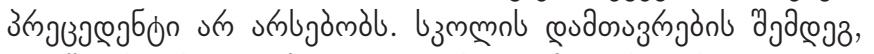

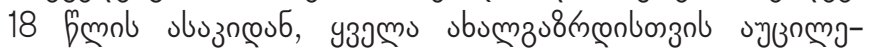

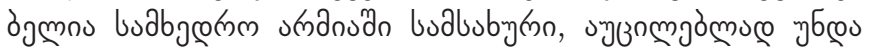

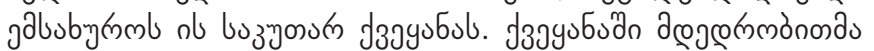

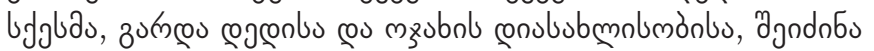

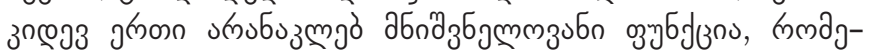

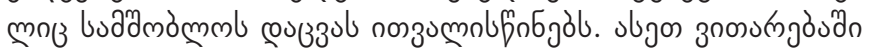

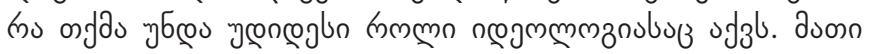

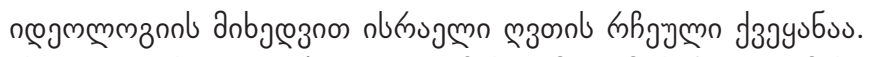

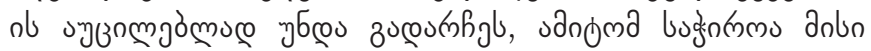

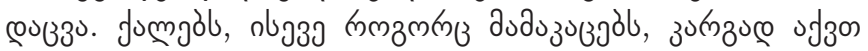

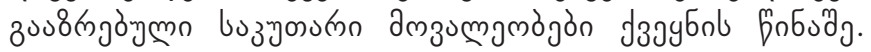

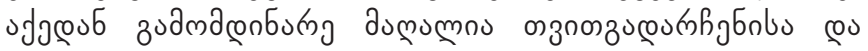

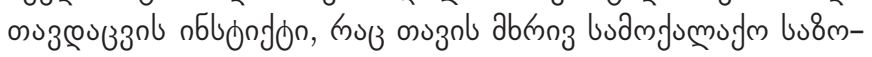

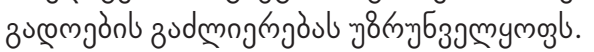

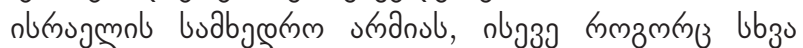

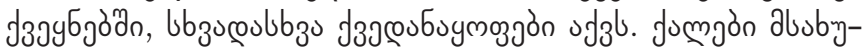

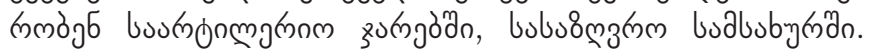

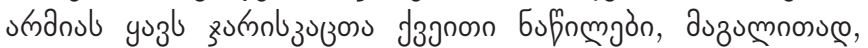

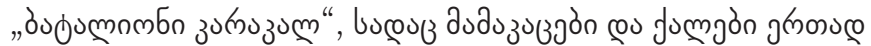

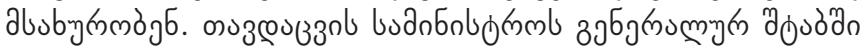

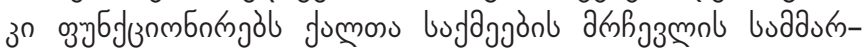

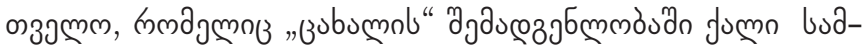

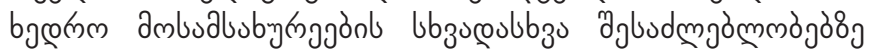
aydumobl.

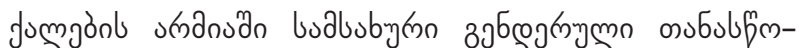

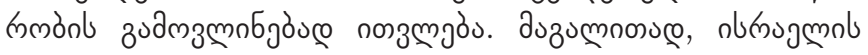

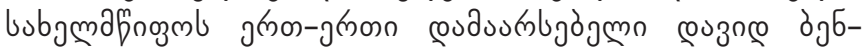

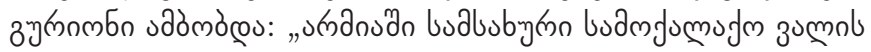

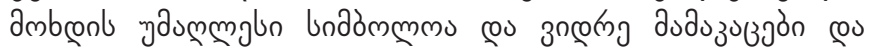

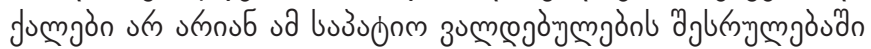

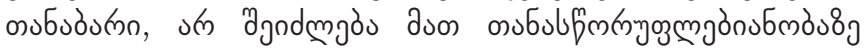

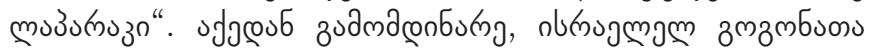

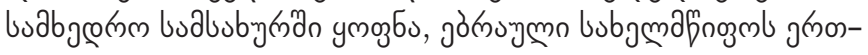
grnon buogigd 3jma zubeos.

numugmals msз

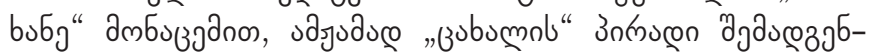

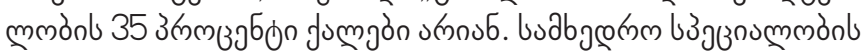

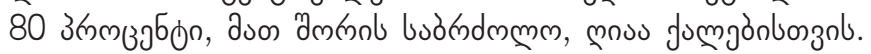

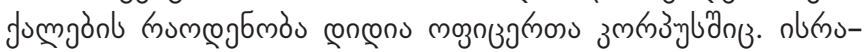

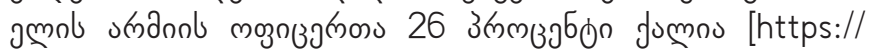
www.facebook.com//sraeliHouseTbilisi/photos / a.352226 934915638.1073741829.352203454917986/442124872592 510].

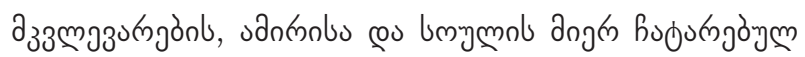

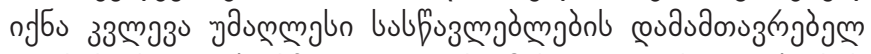

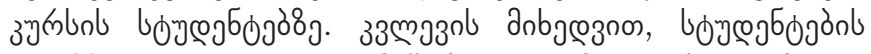

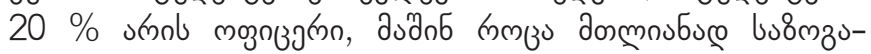

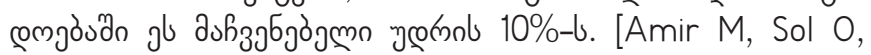
1999.] buzumegàmmm buabgenm buabubymols coubring-

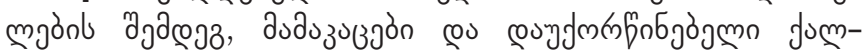

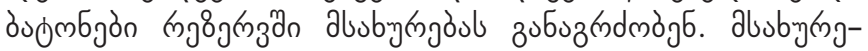

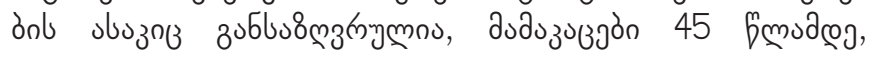


bmmм Jumöudm

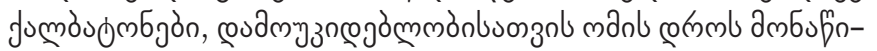

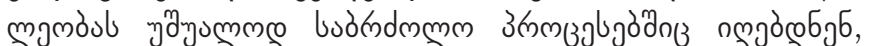

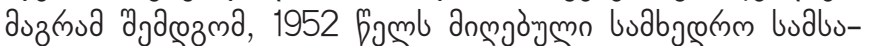

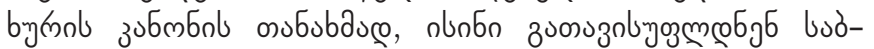

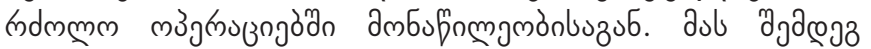

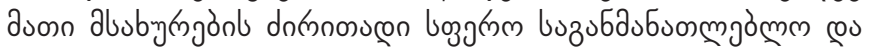

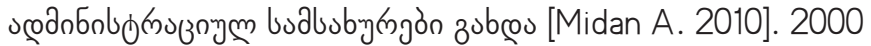

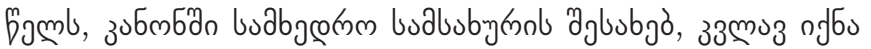

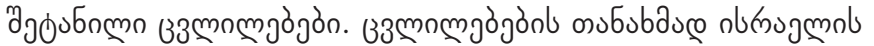

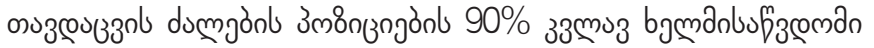

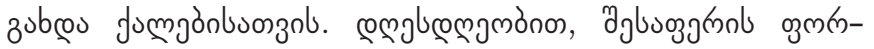

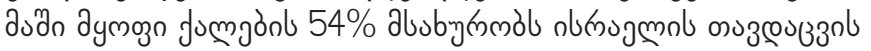

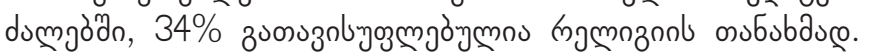

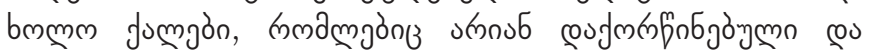

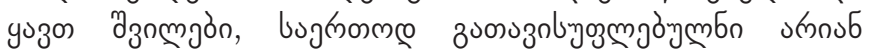

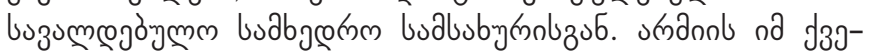

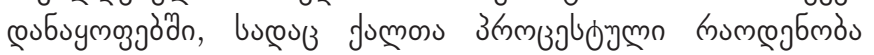

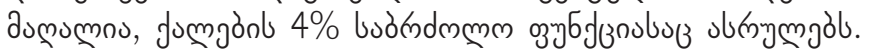

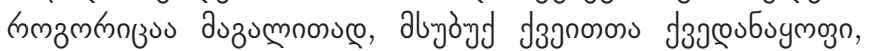

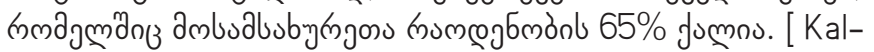
fi-Amir G, 2008].

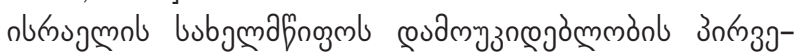

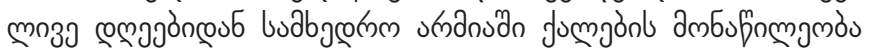

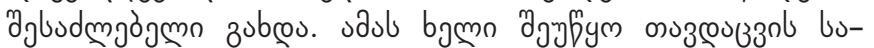

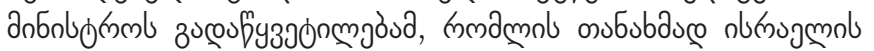

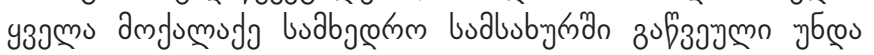

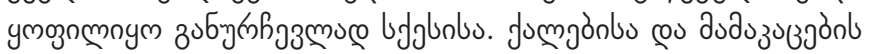

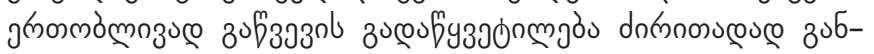

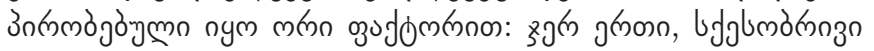

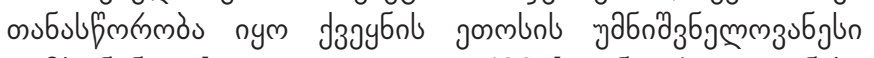

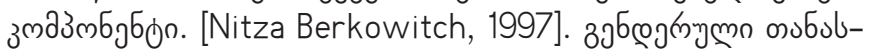

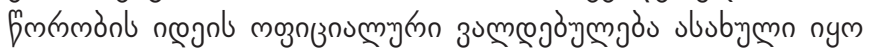

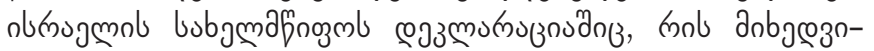

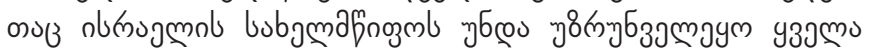

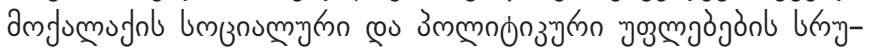

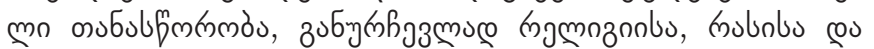
byglobso. The Declaration of the Establishment of the State

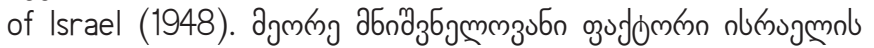

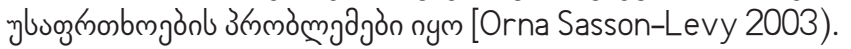

1948 fmnl œ

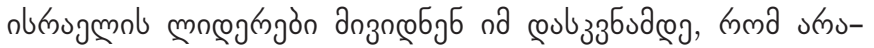

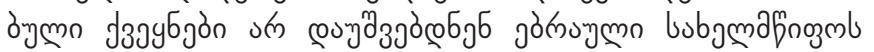

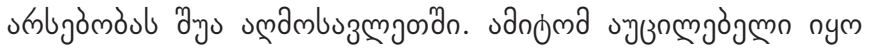

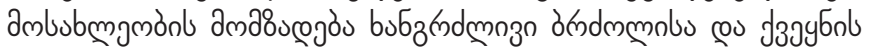

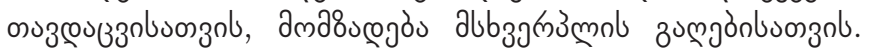
[Joyce Robbins \& Uri Ben-Eliezer, 2000]. sannnzue, bu-

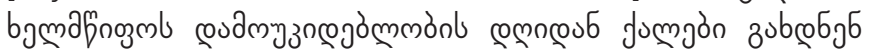

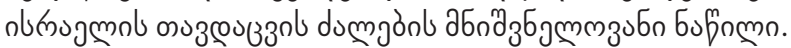

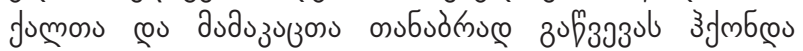

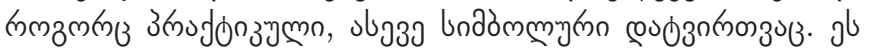

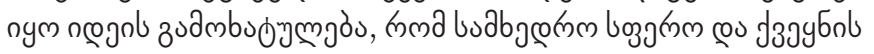

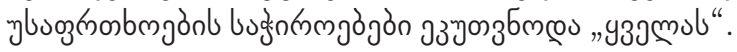

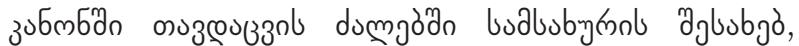

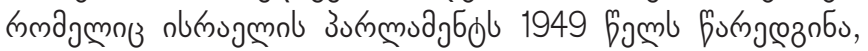

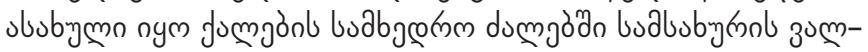

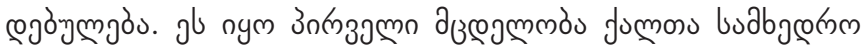

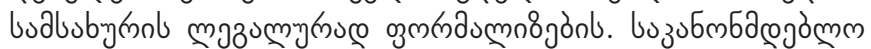

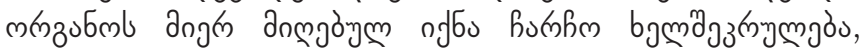

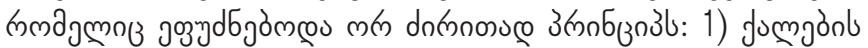

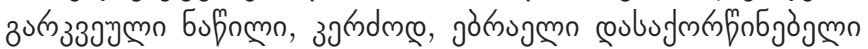

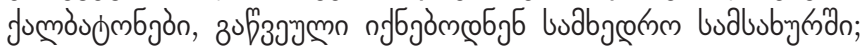

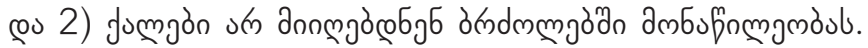

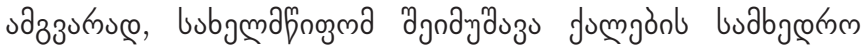

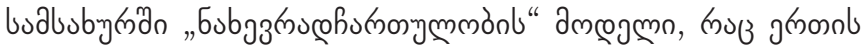

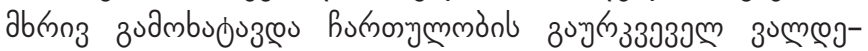

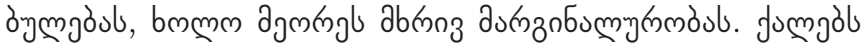

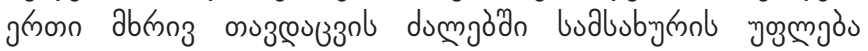

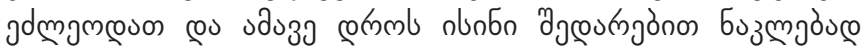

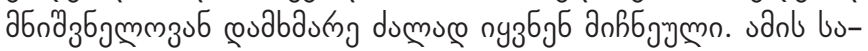

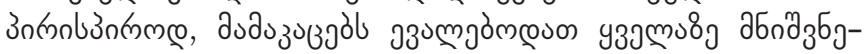

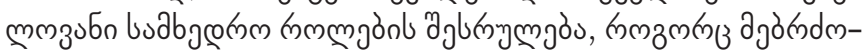

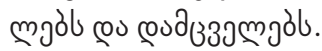

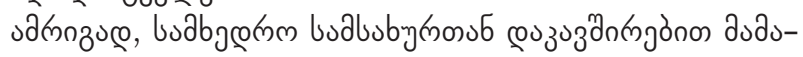

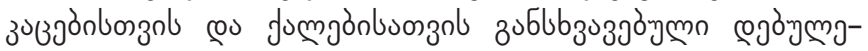
ajö zubnbus

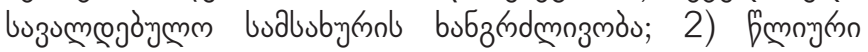

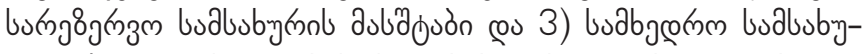

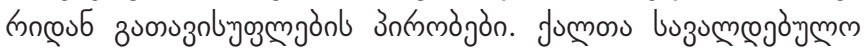

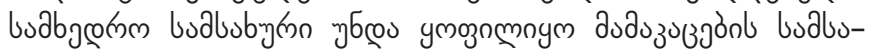

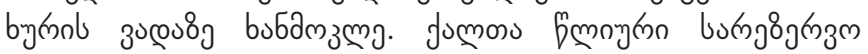

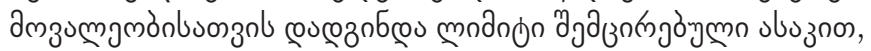

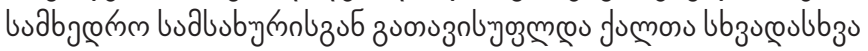
s3yoggòn [Defense Service Law, 5709-1949, 25 LSI 271,

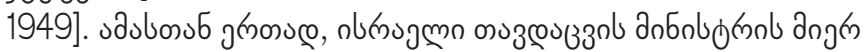

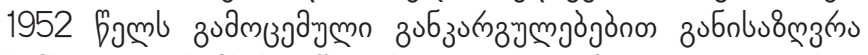

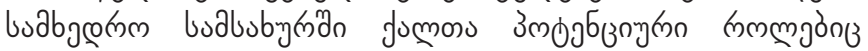
[Defense Service Regulations, 1952].

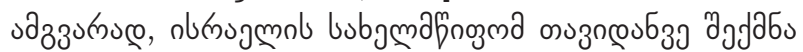

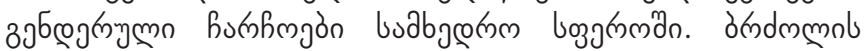

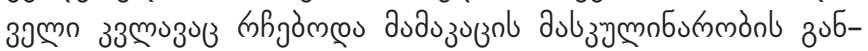

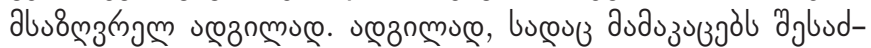

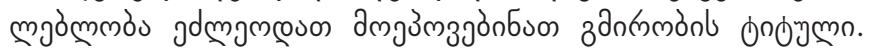

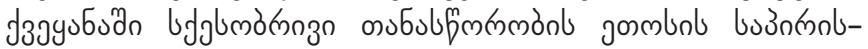

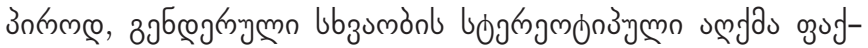

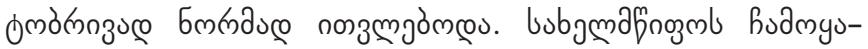

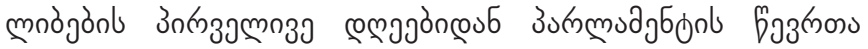

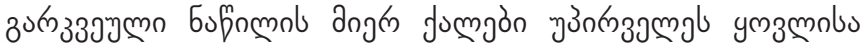

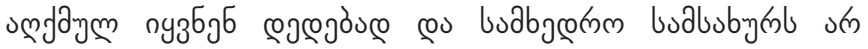

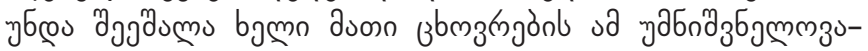

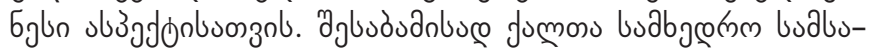

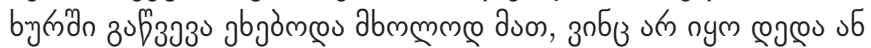

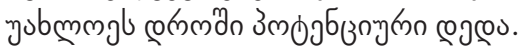

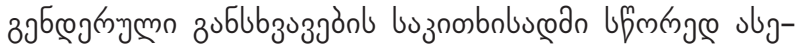

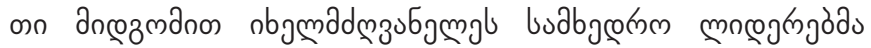

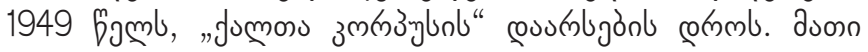




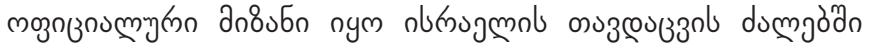

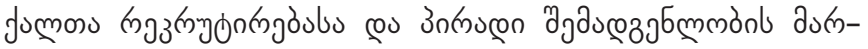

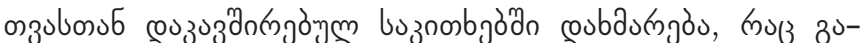

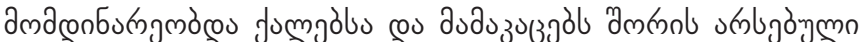

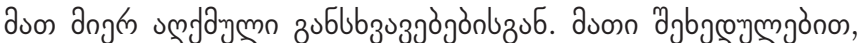

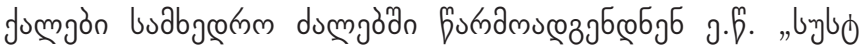

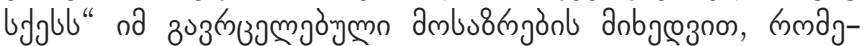

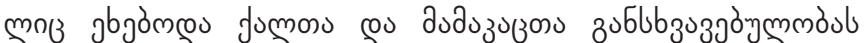

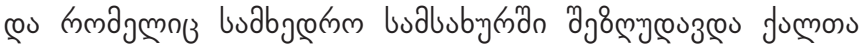

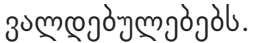

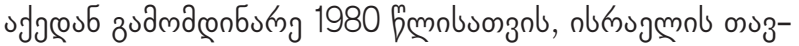

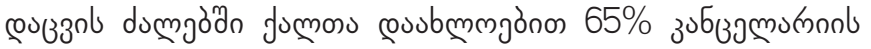

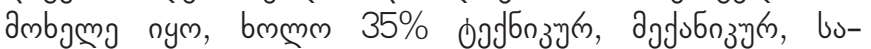

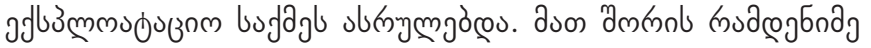

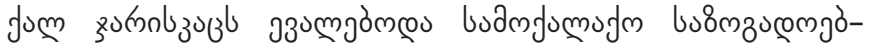

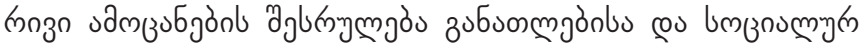
bog ๓nm

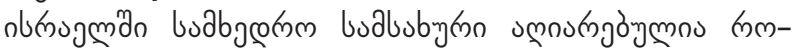

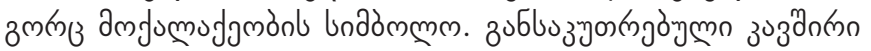

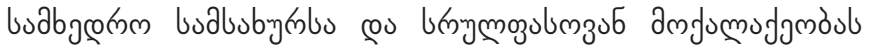

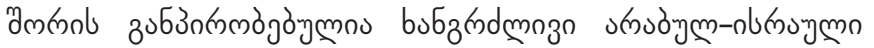

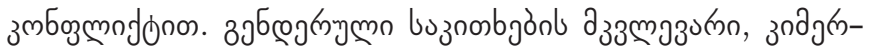

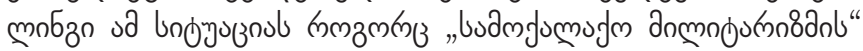

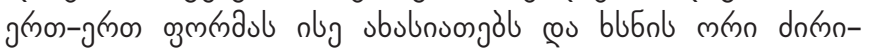

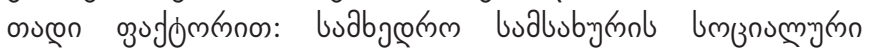

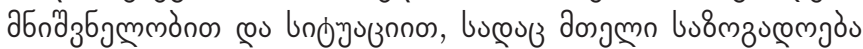

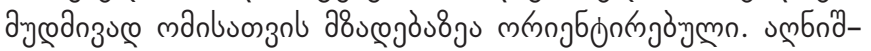

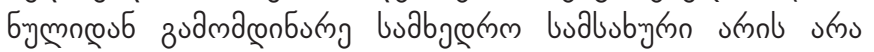

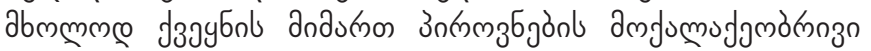

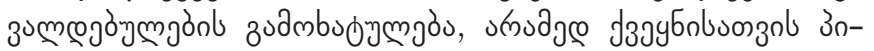

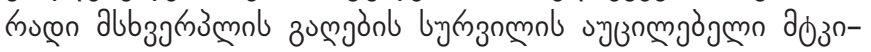

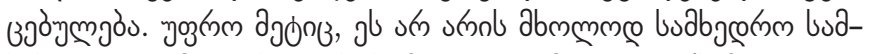

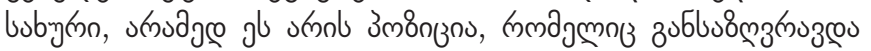

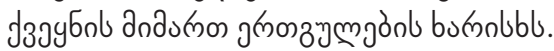

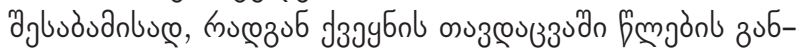

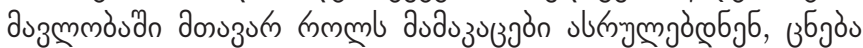

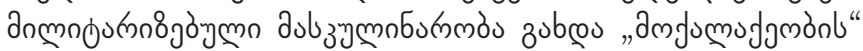

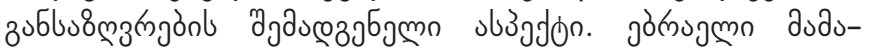

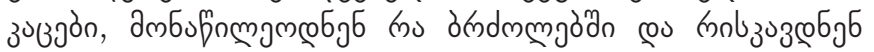

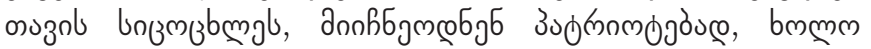

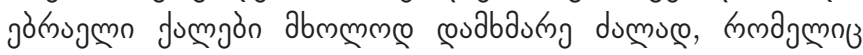

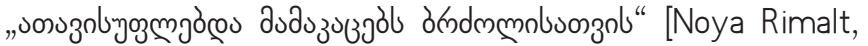

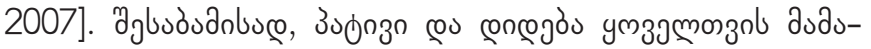

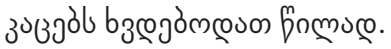

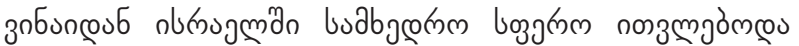

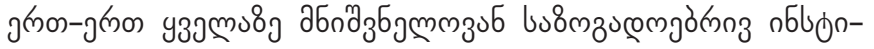

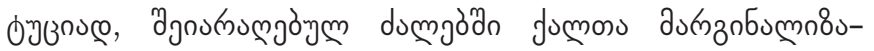

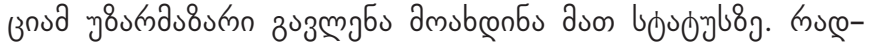

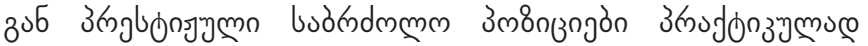

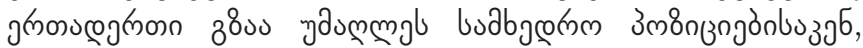

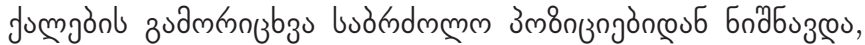

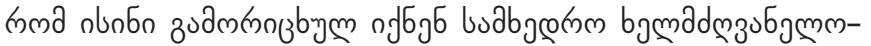

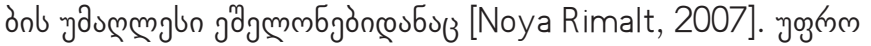

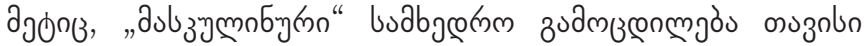

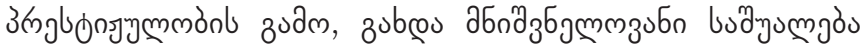

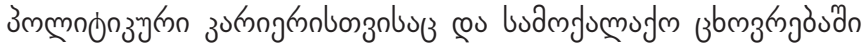

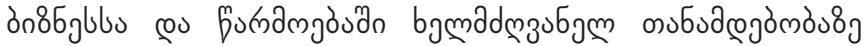

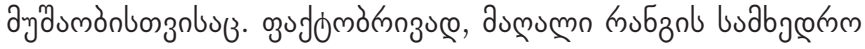

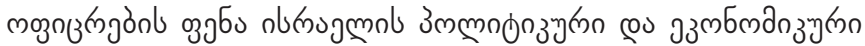

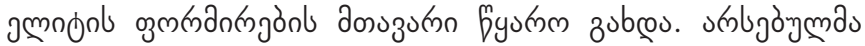

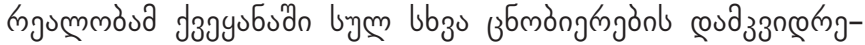

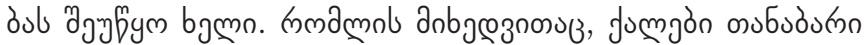

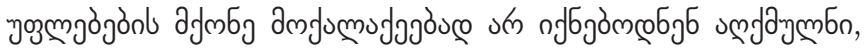

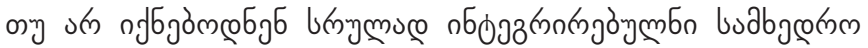

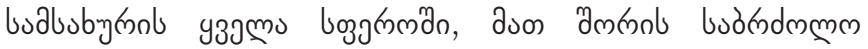

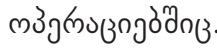

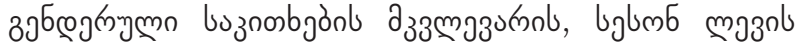

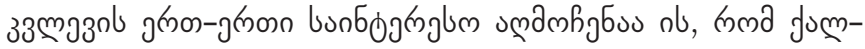

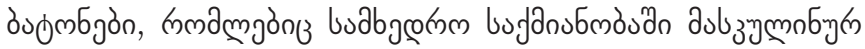

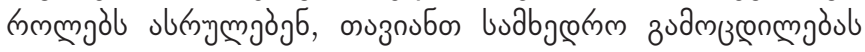

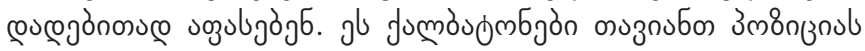

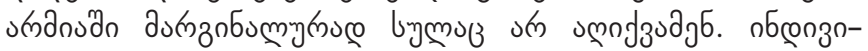

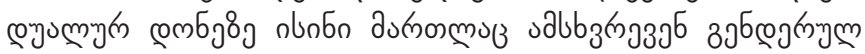

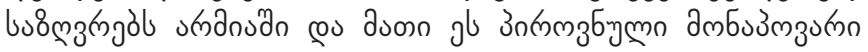

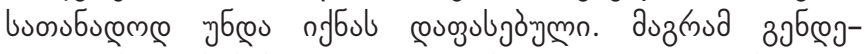

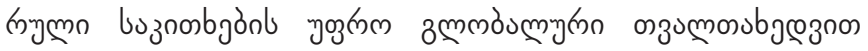

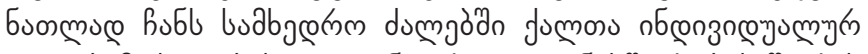

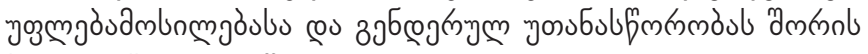

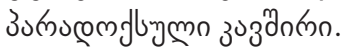

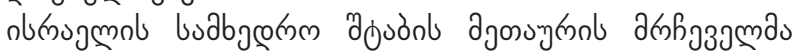

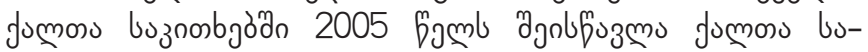

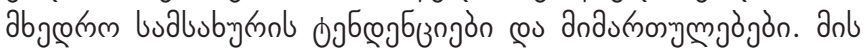

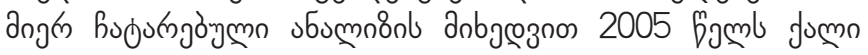

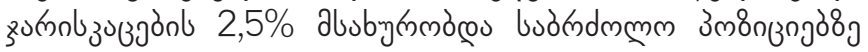

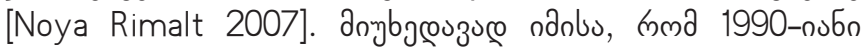

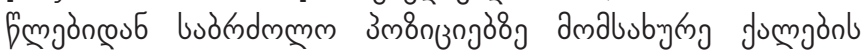

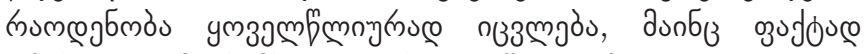

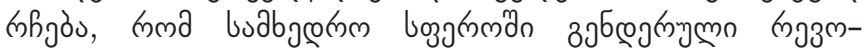

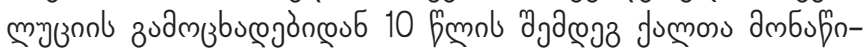

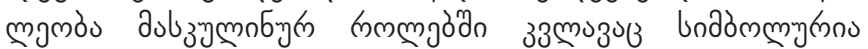

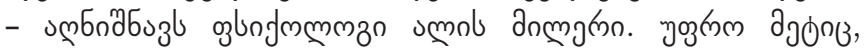

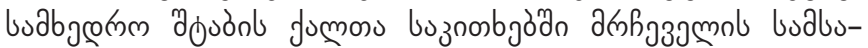

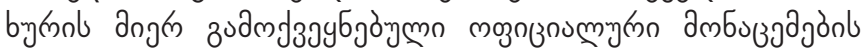

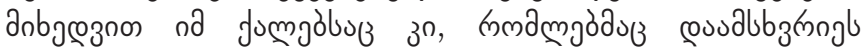

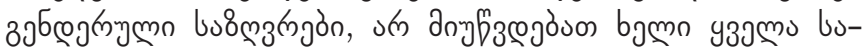

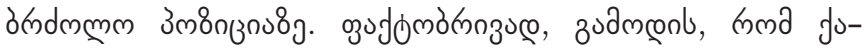

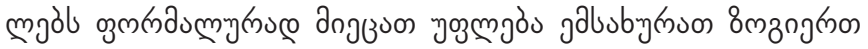

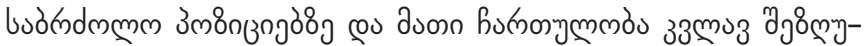

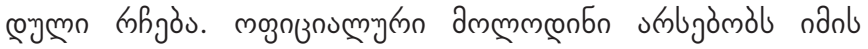

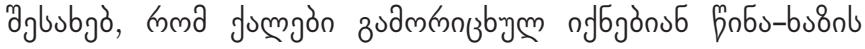

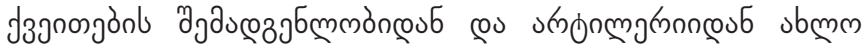

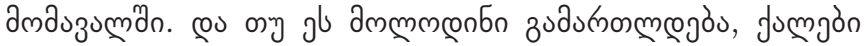

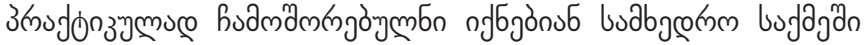
алолу

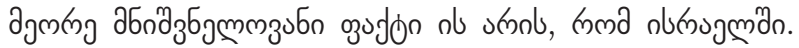

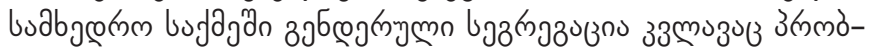




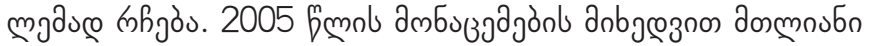

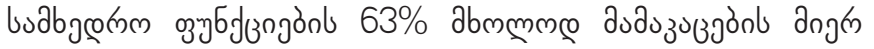

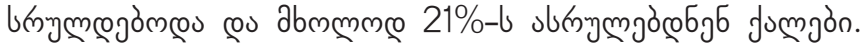

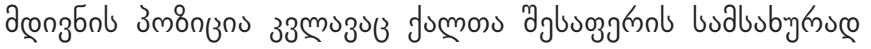

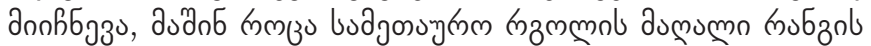

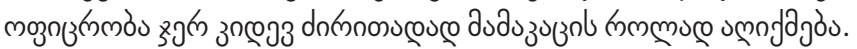

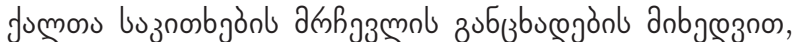

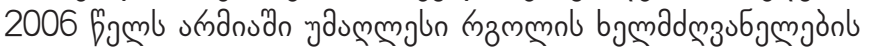

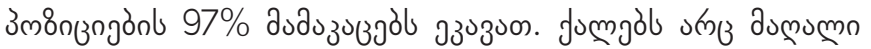

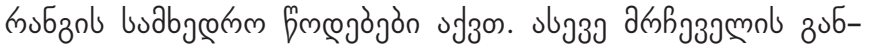

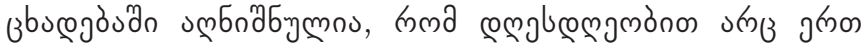
jumb sm u

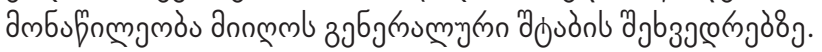

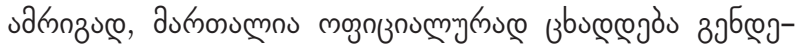

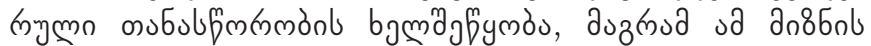

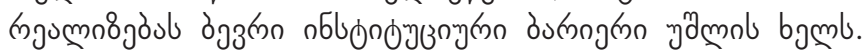

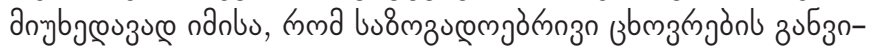

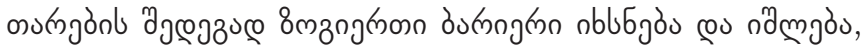

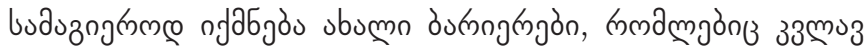

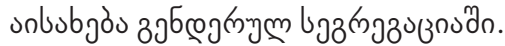

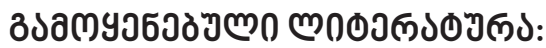

1. Solomon Z, Ginzburg K. The epidemiology of combatinduced post-traumatic stress disorder (PTSD). In: I Levav, editor. Psychiatric and behavioral disorders in Israel: from Epidemiology to Mental Health Action. Jerusalem, New York: Gefen, 2009, pp. 212-232.

2. https://www.facebook.com/IsraeliHouseTbilisi/photos/a.352226934915638. 1073741829.352203454917986/44212487 2592510/ 3noిnonb osknnก 21.12.2017

3. Amir M, Sol O. Psychological impact and prevalence of traumatic events in a student sample in Israel: The effect of multiple traumatic events and physical injury. J Trauma Stress 1999; 12(1): 139-154.

4. Midan A. Israeli women - barely hanging on to the status quo. Special article for Israel's 62 nd Independence Day. Yediot Aharonot. Tel Aviv, 2010, pp. 4-10

5. Kalfi-Amir G. Women's army service in the IDF (Hebrew). Tel Aviv: Office of the Advisor to the Chief of Staff for Women in Israel Defense Forces, Israel Defense Forces, 2008.

6. Sarousi N, El-Peleg D. In the battlefield of the future we will be better than the men: Interview with Gila Kalfi-Amir, Commander of Women's Forces in the Israeli Defence Forces in honor of International Women's Day Bemahaneh (Hebrew) 2010: 31-35.

7. Nitza Berkowitch, Motherhood as a National Mission: The Construction of Womanhood in the Legal Discourse in Israel, 20WOMEN'S STUD. INT'L F. 605, 608 (1997).

8. The Declaration of the Establishment of the State of Israel, 5708-1948, 1 LSI 3 (1948) (Isr.), translation available at http:// www.knesset.gov.il/docs/eng/megilat_eng.htm

9. Orna Sasson-Levy, Feminism and Military Gender Practices: Israeli Women Soldiers in Masculine Roles, 73 SOC. INQUIRY 440, 444 (2003) [hereinafter Sasson-Levy, Feminism and Military Gender Practices].

10. Joyce Robbins \& Uri Ben-Eliezer, "New Roles or “New Times"? Gender Inequality and Militarism in Israel's Nation-in-Arms, 7 SOC. POLITICS: INT'L STUD. GENDER, STATE \& SOC'Y 309, 317 (2000).

11. Defense Service Law, 5709-1949, 25 LSI 271 (1949) (Isr.), art. 11 (allowing exemptions for married women, mothers, pregnant women, religious women, and conscientious objectors, among others).

12. Defense Service Regulations (The Roles of a Woman in Regular [Military] Service), 5712- 1952, 254 KT (Administrative Regulations of Israel) 650 (1952).

13. Noya Rimalt, Women In the Sphere of Masculinity: The Double Edged Sword of Women's Integration in the Military, 2007

14. Halperin-Kaddari, supra note 46 , at 155

15. Dana S. Levin "'You're Always First a Girl": Emerging Adult Women, Gender, and Sexuality in the Israeli Army. Journal of Adolescent Research 26(1) 3-29, 2011 


\section{GENDER ISSUES AND CARACTERISTICS OF ISRAEL ARMY}

ELENE GRATIASHVILI

https://doi.org/10.35945/gb.2017.04.017

Phd Student of St. Andrew the First-Called Georgian University of the Patriarchate of Georgia University, Georgia

\section{KEYWORDS: GENDER ISSUES, GENDER SEGREGATION, GENDER EQUALITY, ISRAEL ARMY}

\section{SUMMARY}

This article covers the characteristics of Israel Army and Gender issues related to it.Israel is the only country, that has obligatory army service for both, men and women. After finishing school, from the age of 18 , it's necessary for everyone to serve in the military. Since the early days of Israel statehood, women have become the part of the Israel Defense Forces. In Israel, military service is recognized as a hallmark of citizenship. While the Jewish male, who served in combat roles, and risked his life, the Jewish woman in her auxiliary roles was only "freeing man for combat".

Although, women were officially allowed to serve in combat roles, their participation is still limited and Gender segregation remains to be a problem. In the end, gender equality should be promoted for solving the existing problems, that would lead to necessary changes in the Armed Forces. 